\title{
Evaluating Collaboration Ability of R\&D Team Members: A Multi-Granular Hesitant Fuzzy Linguistic Approach
}

\author{
Zhen Zhang $^{a}$ and Junliang Gao ${ }^{a}$ and Wenyu $\mathbf{Y u}^{b *}$ \\ ${ }^{a}$ Institute of Systems Engineering, Dalian University of Technology, Dalian, China \\ Emails: zhen.zhang@dlut.edu.cn; jlgao@mail.dlut.edu.cn \\ ${ }^{b}$ School of Economics and Management, Dalian University of Technology, Dalian, China \\ Email: ywyeva@mail.dlut.edu.cn
}

\begin{abstract}
Evaluating each member's collaboration ability in an R\&D team is of vital importance for senior managers to improve the team performance for R\&D activities. Due to the difference in knowledge and education background, each team member usually tends to use multi-granular hesitant fuzzy linguistic term sets to elicit their assessments over team members who have working relationship with him/her with respect to each criterion. In this paper, a multi-granular hesitant fuzzy linguistic approach is developed to evaluate the collaboration ability of R\&D team members. In the proposed approach, all the individual multi-granular hesitant fuzzy linguistic assessments provided by each team member with respect to each criterion are first transformed into their fuzzy envelopes and then aggregated to obtain the overall assessment of each team member's collaboration ability, which are in the form of trapezoidal fuzzy numbers. By ranking the trapezoidal fuzzy numbers, the collaboration ability of team members can be ranked. The evaluation values are also transformed into multigranular hesitant fuzzy linguistic term sets to provide interpretable results about each team member's collaboration ability. Finally, a numerical example is presented to illustrate the proposed approach.
\end{abstract}

Keywords: Collaboration ability evaluation, R\&D, Hesitant fuzzy linguistic term set.

\section{Introduction}

With the rapid development of global competition, R\&D activities have been more and more important for companies and other organizations, which are of- ten participated by $R \& D$ teams to cope with rapid change of the world [8]. An R\&D team is a group of R\&D personnel who take responsibility for scientific and technological research and development. Most of the $R \& D$ teams exist in high-tech industry and are composed of professionals from different fields of work for some certain purposes.

An R\&D project usually needs joint efforts and cooperation of team members with different professional skills [9]. A good and effective combination of R\&D team members enables the team to better use knowledge and information related to $\mathrm{R} \& \mathrm{D}$, which will also inspire new and diverse views, ideas of team members [22]. Perfect collaboration between team members will promote person-to-person interaction, which can help improve mutual satisfaction among members and team performance eventually. Therefore, it is of vital importance to evaluate collaboration ability of team members so that the team leader could always know the cooperative situation and make strategic adjustments in a timely manner.

In the literature, team collaboration has been investigated from different perspectives. For instance, Son and Rojasan [16] proposed an agent-based modelling and simulation approach to analyze the evolution of collaboration in temporary project teams. Defranco et al. [1] developed a cognitive collaborative model to improve the performance in engineering teams. MacBryde and Mendibil [11] discussed the design of performance measurement systems for a team and pointed out that team member satisfaction is an important dimension for performance measurement. Fan et al. [4] used a 2-tuple fuzzy linguistic approach to evaluate the collaboration satisfaction of an NPD team based on mutual-evaluation of team members. Based on Fan et al's work, Zhang et al. [26] proposed an approach to evaluate the collaborative satisfaction for project management team in integrated project delivery mode.

Previous studies have significantly advanced the study 
of team collaboration evaluation. One can find that fuzzy linguistic approaches are useful tools for team collaboration evaluation due to the fact that collaboration ability is subjective experience of team members which can be assessed using linguistic variables to depict the fuzziness and imprecise knowledge of team members [14]. However, existing approaches are not suitable to directly deal with practical R\&D team collaboration ability evaluation problems for the following reasons. First, existing approaches usually assumed that team members can only use a simple linguistic term to elicit assessments over other team members' collaboration ability, which cannot deal with the situations when team members may have some hesitancy among some linguistic terms. The hesitant fuzzy linguistic term set (HFLTS) which allows decision makers to use some consecutive linguistic terms can be an effective tool for team members to elicit linguistic assessments flexibly $[13,21,17]$. Second, due to differences in knowledge background and culture, different team members tend to use linguistic term sets with different granularities [12, 20, 28, 25]. However, these situations have not been fully considered in existing studies.

To overcome the above limitations, the aim of this paper is to develop a multi-granular hesitant fuzzy linguistic approach to evaluate collaboration ability of R\&D team members, in which multi-granular HFLTSs are used by team members to provide mutualevaluations over other team members and the fuzzy envelopes of HFLTSs are used to transform and aggregate multi-granular hesitant fuzzy linguistic assessments, based on which the collaboration ability of R\&D team members can be evaluated and ranked. The evaluation results are also transformed into HFLTSs defined on the linguistic term sets used by different team members to provide interpretable results about each team member's collaboration ability.

The rest of the paper is organized as follows. Section 2 presents preliminaries on trapezoidal fuzzy numbers (TFNs) and HFLTSs. In section 3, an approach is developed to evaluate collaboration ability of $R \& D$ team members. In section 4, a numerical example is utilized to demonstrate the proposed method. Finally, some conclusions are drawn in section 5 .

\section{Preliminaries}

In this section, some basic knowledge about TFNs and HFLTSs is introduced.

\section{$2.1 \quad$ TFNs}

Zadeh [24] proposed the concept of fuzzy sets to depict the uncertainty in human beings' vague and imprecise knowledge.

Definition 1. [24] Let $X$ be a universe set. A fuzzy set $A$ in $X$ is characterized by a membership function $\mu_{A}(x)$ which associates each $x$ in $X$ with a real number in interval $[0,1]$. The function $\mu_{A}(x)$ denotes the membership degree of $x$ in $A$.

TFN is one of the most widely used fuzzy sets, which is defined below.

Definition 2. [7] Let $A$ be a fuzzy set in $X$, then $A=T(a, b, c, d)$ is a TFN if its membership function $\mu_{A}(x)$ is given by

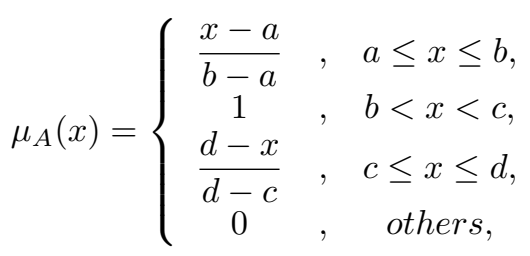

where $a, b, c, d$ are real numbers.

Given two TFNs $A_{1}=T\left(a_{1}, b_{1}, c_{1}, d_{1}\right), \quad A_{2}=$ $T\left(a_{2}, b_{2}, c_{2}, d_{2}\right)$, some operations can be defined as follows [7].

(1) $A_{1}+A_{2}=T\left(a_{1}+a_{2}, b_{1}+b_{2}, c_{1}+c_{2}, d_{1}+d_{2}\right)$;

(2) $k A_{1}=T\left(k a_{1}, k b_{1}, k c_{1}, k d_{1}\right)$.

In addition, the Hamming distance between two TFNs $A_{1}=T\left(a_{1}, b_{1}, c_{1}, d_{1}\right)$ and $A_{2}=T\left(a_{2}, b_{2}, c_{2}, d_{2}\right)$ is defined as

$d\left(A_{1}, A_{2}\right)=\frac{1}{4}\left(\left|a_{1}-a_{2}\right|+\left|b_{1}-b_{2}\right|+\left|c_{1}-c_{2}\right|+\left|d_{1}-d_{2}\right|\right)$

In [19], it is defined the centroid formulae of an TFN.

Definition 3. [19] Let $A=T(a, b, c, d)$ be a TFN, then the centroid of $A$ can be calculated as $\left(\bar{x}_{A}, \bar{y}_{A}\right)$, where

$$
\begin{aligned}
& \bar{x}_{A}=\frac{1}{3}\left(a+b+c+d-\frac{c \times d-a \times b}{(c+d)-(a+b)}\right) \\
& \bar{y}_{A}=\frac{1}{3}\left(1+\frac{c-b}{(c+d)-(a+b)}\right) .
\end{aligned}
$$

Based on the centroid formulae of TFNs, a comparison rule is proposed to compare and rank TFNs [18]. Let $A_{1}=T\left(a_{1}, b_{1}, c_{1}, d_{1}\right)$ and $A_{2}=T\left(a_{2}, b_{2}, c_{2}, d_{2}\right)$ be two TFNs, then

(a) If $\bar{x}_{A_{1}}>\bar{x}_{A_{2}}$, then $A_{1} \succ A_{2}$;

(b) If $\bar{x}_{A_{1}}<\bar{x}_{A_{2}}$, then $A_{1} \prec A_{2}$;

(c) If $\bar{x}_{A_{1}}=\bar{x}_{A_{2}}$ and $\bar{y}_{A_{1}}>\bar{y}_{A_{2}}$, then $A_{1} \succ A_{2}$; If $\bar{x}_{A_{1}}=\bar{x}_{A_{2}}$ and $\bar{y}_{A_{1}}<\bar{y}_{A_{2}}$, then $A_{1} \prec A_{2}$; If $\bar{x}_{A_{1}}=\bar{x}_{A_{2}}$ and $\bar{y}_{A_{1}}=\bar{y}_{A_{2}}$, then $A_{1} \sim A_{2}$. 


\subsection{Hesitant fuzzy linguistic term sets}

In this section, we introduce some knowledge related to HFLTSs, including the concept and the fuzzy envelope of an HFLTS.

Let $S=\left\{s_{0}, s_{1}, \ldots, s_{g}\right\}$ be a linguistic term set with odd cardinality, where element $s_{i}$ denotes the $i$-th linguistic term in $S$ and $g+1$ is the granularity of $S$. A linguistic term set $S$ satisfies the following characteristics[5, 15]: (1) The set is ordered: $s_{i}>s_{j}$, if $i>j$; (2) A negation operator $N e g\left(s_{i}\right)=s_{j}$, such that $j=g-i$; (3) A maximization operator and a minimization operator: $\max \left(s_{i}, s_{j}\right)=s_{i}, \min \left(s_{i}, s_{j}\right)=s_{j}$, if $i>j$.

For decision making problems with high level uncertainty and complexity, decision makers may hesitate among some linguistic terms and use more than one linguistic terms. Rodríguez et al. [13] defined the concept of HFLTSs to characterize such situations.

Definition 4. [13] Let $S=\left\{s_{0}, s_{1}, \ldots, s_{g}\right\}$ be a linguistic term set. An HFLTS $H^{S}$ on $S$ is an ordered finite subset of consecutive linguistic term sets in $S$.

To carry out the computing with words processes, the fuzzy envelope of an HFLTS is proposed in [10], which represents an HFLTS using a TFN obtained by the aggregation of the linguistic terms that compound the HFLTS.

Definition 5. [10] Let $S=\left\{s_{0}, s_{1}, \ldots, s_{g}\right\}$ be a linguistic term set and $H^{S}=\left\{s_{i}, \ldots, s_{j}\right\}$ be an HFLTS defined on $S$ such that $s_{k} \in S, k=i, i+1, \ldots, j$. The fuzzy envelope $\operatorname{env}_{F}\left(H^{S}\right)$ of $H^{S}$ can be defined as a TFN $T(a, b, c, d)$, denoted by

$$
\operatorname{env}_{F}\left(H^{S}\right)=T(a, b, c, d) .
$$

Details about how to calculate the fuzzy envelope of an HFLTS can be found in [10].

\section{The proposed approach}

In this section, an approach is developed to evaluate collaboration ability of R\&D team members. First of all, the formulation of the collaboration ability evaluation problem is presented.

Let $P=\left\{P_{1}, P_{2}, \ldots, P_{n}\right\}$ be the set of team members in an R\&D team, where $P_{i}$ is the $i$-th team member, $i \in I=\{1,2, \ldots, n\}$. In order to evaluate collaboration ability of team members, each team member is requested to provide his/her evaluation over other team members who have working relationship with him/her with respect to some criteria. Let $C=$ $\left\{C_{1}, C_{2}, \ldots, C_{m}\right\}$ be the set of criteria concerning collaboration ability, where $C_{k}$ denotes the $k$-th criterion, $k \in K=\{1,2, \ldots, m\}$, and $w=\left(w_{1}, w_{2}, \ldots, w_{m}\right)^{\mathrm{T}}$ denotes the weight vector of the criteria such that $\sum_{k=1}^{m} w_{k}=1, w_{k} \geq 0, k \in K$.

The working relationship between team members in the R\&D team is denoted by a matrix $R=\left(r_{i j}\right)_{n \times n}$, where $r_{i j}$ represents the working relationship between team members $P_{i}$ and $P_{j}$. In particular, $r_{i j}=1$ indicates that there exists working relationship between $P_{i}$ and $P_{j}, r_{i j}=0$ means there is no working relationship between $P_{i}$ and $P_{j}$, and $r_{i i}=0$ means that $P_{i}$ has no working relationship with himself/herself, $i, j \in I$. Note that the elements in each row and column of $R$ cannot be zero at the same time, i.e., the following conditions $\sum_{j=1}^{n} r_{i j} \neq 0, i \in I$ and $\sum_{i=1}^{n} r_{i j} \neq 0, j \in I$ should be satisfied. Otherwise, the team will be divided into two or more sub-teams [4].

For convenience, let the linguistic term set used by the $i$-th team member be $S^{g(i)+1}=$ $\left\{s_{0}^{g(i)+1}, s_{1}^{g(i)+1}, \ldots, s_{g(i)}^{g(i)+1}\right\}$, where $g(i)+1$ is the granularity of $S^{g(i)+1}, i \in I$, and the evaluation over team member $P_{j}$ provided by $P_{i}$ with respect to the $k$-th criterion concerning collaboration ability be $h_{i j}^{k}$, $i, j \in I, k \in K$. If $r_{i j}=1$, then $h_{i j}^{k} \in H^{S^{g(i)+1}}$, where $H^{S^{g(i)+1}}$ denotes the set of all the HFLTSs defined on $S^{g(i)+1}$; If $r_{i j}=0$, let $h_{i j}^{k}=-, i, j \in I, k \in K$. Therefore, an evaluation matrix $H^{k}$ with respect to $C_{k}$ can be denoted by

$$
H^{k}=\left(h_{i j}^{k}\right)_{n \times n}=\left(\begin{array}{cccc}
h_{11}^{k} & h_{12}^{k} & \cdots & h_{1 n}^{k} \\
h_{21}^{k} & h_{22}^{k} & \cdots & h_{2 n}^{k} \\
\vdots & \vdots & \ddots & \vdots \\
h_{n 1}^{k} & h_{n 2}^{k} & \cdots & h_{n n}^{k}
\end{array}\right), k \in K
$$

Based on the evaluation matrices $H^{1}, H^{2}, \ldots, H^{m}$, the following procedure is proposed to evaluate collaboration ability of R\&D team members.

First of all, all the HFLTSs in each $H^{k}$ are transformed into their corresponding fuzzy envelopes. Let $y_{i j}^{k}=$ $T\left(y_{i j, 1}^{k}, y_{i j, 2}^{k}, y_{i j, 3}^{k}, y_{i j, 4}^{k}\right)$ be the fuzzy envelope of $h_{i j}^{k}$, $\forall r_{i j}=1, i, j \in I, k \in K$, then we have

$$
\begin{aligned}
T\left(y_{i j, 1}^{k}, y_{i j, 2}^{k}, y_{i j, 3}^{k}, y_{i j, 4}^{k}\right) & =\operatorname{env}_{F}\left(h_{i j}^{k}\right), \\
& \forall r_{i j}=1, i, j \in I, k \in K .
\end{aligned}
$$

For the convenience of calculation, let $y_{i j}^{k}=$ $T\left(y_{i j, 1}^{k}, y_{i j, 2}^{k}, y_{i j, 3}^{k}, y_{i j, 4}^{k}\right)=T(0,0,0,0), \forall r_{i j}=0, i, j \in$ $I, k \in K$.

Accordingly, each hesitant fuzzy linguistic matrix $H^{k}$ can be transformed into a TFN evaluation matrix as $Y^{k}=\left(y_{i j}^{k}\right)_{n \times n}, i, j \in I, k \in K$.

By aggregating all TFN evaluation matrices $Y^{1}, Y^{2}, \ldots, Y^{m}, \quad$ a collective mutual-evaluation 
matrix $Y=\left(y_{i j}\right)_{n \times n}$ can be obtained where

$$
\begin{array}{r}
y_{i j}=T\left(y_{i j, 1}, y_{i j, 2}, y_{i j, 3}, y_{i j, 4}\right)=\oplus_{k=1}^{m} w_{k} y_{i j}^{k} \\
=T\left(\sum_{k=1}^{m} w_{k} y_{i j, 1}^{k}, \sum_{k=1}^{m} w_{k} y_{i j, 2}^{k}, \sum_{k=1}^{m} w_{k} y_{i j, 3}^{k}, \sum_{k=1}^{m} w_{k} y_{i j, 4}^{k}\right) \\
\forall r_{i j}=1, i, j \in I
\end{array}
$$

and

$$
\begin{aligned}
y_{i j}=T\left(y_{i j, 1}, y_{i j, 2}, y_{i j, 3}, y_{i j, 4}\right)=T(0,0,0,0), & \\
& \forall r_{i j}=0, i, j \in I .
\end{aligned}
$$

Based on the collective mutual-evaluation matrix $Y$, the evaluation value of the $i$-th team member's collaboration ability, denoted by $y_{i}$, can be calculated by aggregating the TFNs of team members who have working relationship with him/her, i.e.,

$$
\begin{aligned}
y_{i} & =T\left(y_{i, 1}, y_{i, 2}, y_{i, 3}, y_{i, 4}\right) \\
& =\left(\frac{\sum_{j=1}^{n} r_{j i} y_{j i, 1}}{\sum_{j=1}^{n} r_{j i}}, \frac{\sum_{j=1}^{n} r_{j i} y_{j i, 2}}{\sum_{j=1}^{n} r_{j i}},\right. \\
& \left.\frac{\sum_{j=1}^{n} r_{j i} y_{j i, 3}}{\sum_{j=1}^{n} r_{j i}}, \frac{\sum_{j=1}^{n} r_{j i} y_{j i, 4}}{\sum_{j=1}^{n} r_{j i}}\right), i \in I .
\end{aligned}
$$

According to the evaluation value of each team member's collaboration ability $y_{i}$, the collaboration ability of each team member can be ranked by the centroidbased ranking rule of fuzzy numbers, see Eq. (3) and the ranking rule below Definition 3.

To help team members know their collaboration ability with others, it is necessary to return the evaluation results to them. However, the evaluation results are in the form of TFNs which are difficult for team members to understand. In this paper, the TFNs are further transformed into HFLTSs defined on the linguistic term sets used by each team member using some approximation operations to provide interpretable results.

Let $N^{i}$ be the maximum number of linguistic terms used by the $i$-th team member to elicit HFLTSs, then the set of all the possible HFLTSs used by the $i$-th team member, denoted by $F_{N^{i}}^{i}$, can be obtained as

$$
F_{N^{i}}^{i}=\left\{l \in H^{S^{g(i)+1}} \mid \# l \leq N^{i}\right\}
$$

For each HFLTS in $F_{N^{i}}^{i}$, its corresponding fuzzy envelope can be calculated as well. For convenience, let $F_{N^{i}}^{i}=\left\{l_{1}, l_{2}, \ldots, l_{\# F_{N^{i}}}\right\}$, and the set of the fuzzy envelopes of HFLTSs in $F_{N^{i}}^{i}$ be $\left\{\operatorname{env}_{F}\left(l_{1}\right), \operatorname{env}_{F}\left(l_{2}\right), \ldots, \operatorname{env}_{F}\left(l_{\# F_{N i}^{i}}\right)\right\}$, where $e n v_{F}\left(l_{q}\right)$ is a TFN calculated by Eq. (4), $q=1,2, \ldots, \# F_{N^{i}}^{i}$. Thus, the HFLTS $\widetilde{y}_{i}$ whose fuzzy envelope has the least distance from $y_{i}$ can be calculated by

$$
\widetilde{y}_{i}=\arg \min _{l_{q} \in F_{N^{i}}^{i}} d\left(y_{i}, \operatorname{env}_{F}\left(l_{q}\right)\right), i \in I .
$$

Obviously, $\widetilde{y}_{i}$ is an HFLTS defined on $S^{g(i)+1}$, which can be easily understood by the team member $P_{i}, i \in$ I.

The procedure to evaluate collaboration ability of $\mathrm{R} \& \mathrm{D}$ team members is demonstrated in Figure 1.

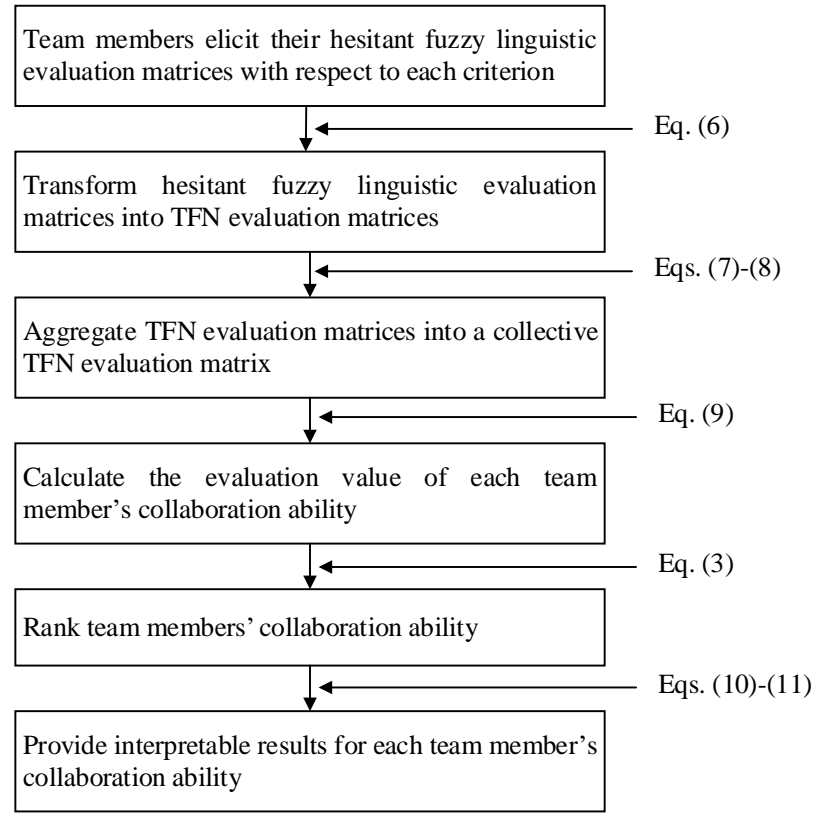

Figure 1: The flowchart of the proposed approach

\section{Illustrative example}

In this section, an example is provided to demonstrate the proposed approach.

A senior manager in a company intends to learn about the collaboration ability of six team members $\left(P_{1}, P_{2}, \ldots, P_{6}\right)$ in an R\&D team in order to improve team performance. When performing the evaluation, five criteria $\left(C_{1}, C_{2}, \ldots, C_{5}\right)$ are considered and the weight vector of the criteria is $w=$ $(0.15,0.2,0.1,0.25,0.3)^{\mathrm{T}}$. Due to differences in culture and knowledge background, multi-granular HFLTSs are provided by each team member to elicit his/her evaluation over team members who have working relationship with him/her. Specifically, $P_{2}$ and $P_{4}$ use the linguistic term set $S^{5}=\left\{s_{0}^{5}\right.$ : poor, $s_{1}^{5}$ : slightly poor, $s_{2}^{5}$ : fair, $s_{3}^{5}$ : slightly good, $\left.s_{4}^{5}: \operatorname{good}\right\}$, $P_{3}$ and $P_{5}$ use the linguistic term set $S^{7}=$ $\left\{s_{0}^{7}:\right.$ very poor, $s_{1}^{7}:$ poor, $s_{2}^{7}$ : slightly poor, $s_{3}^{7}$ : 
fair, $s_{4}^{5}:$ slightly good, $\left.s_{5}^{7}: \operatorname{good}, s_{6}^{7}: \operatorname{very} \operatorname{good}\right\}$, and $P_{1}$ and $P_{6}$ use the linguistic term set $S^{9}=$ $\left\{s_{0}^{9}\right.$ : extremely poor, $s_{1}^{9}$ : very poor, $s_{2}^{9}$ : poor, $s_{3}^{9}$ : slightly poor, $s_{4}^{9}$ : fair, $s_{5}^{9}$ : slightly good, $s_{6}^{9}: \operatorname{good}, s_{7}^{9}$ : very good, $s_{8}^{9}$ : extremely good $\}$.

The working relationship between the six members in the team is described by a matrix as follows:

$$
R=\left[\begin{array}{llllll}
0 & 1 & 1 & 1 & 1 & 1 \\
1 & 0 & 1 & 0 & 1 & 1 \\
1 & 1 & 0 & 1 & 0 & 0 \\
1 & 0 & 1 & 0 & 1 & 1 \\
1 & 1 & 0 & 1 & 0 & 1 \\
1 & 1 & 0 & 1 & 1 & 0
\end{array}\right]
$$

Each team member provides his/her evaluation based on working relationship, and the multi-granular hesitant fuzzy linguistic evaluation matrices with respect to the five criteria are shown as follows. $H^{1}=$

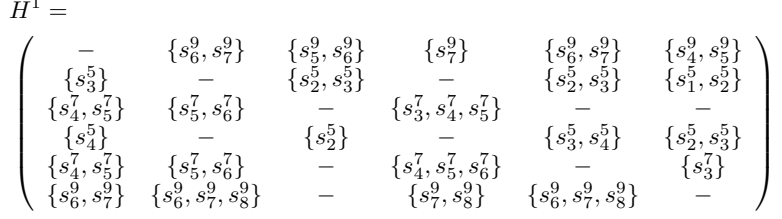

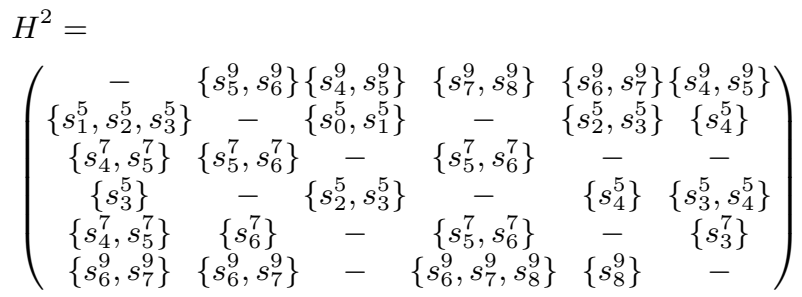$$
H^{3}=
$$$$
\left(\begin{array}{cccccc}
- & \left\{s_{5}^{9}, s_{6}^{9}, s_{7}^{9}\right\} & \left\{s_{6}^{9}\right\} & \left\{s_{5}^{9}, s_{6}^{9}\right\} & \left\{s_{6}^{9}\right\} & \left\{s_{5}^{9}, s_{6}^{9}\right\} \\
\left\{s_{2}^{5}, s_{3}^{5}, s_{4}^{5}\right\} & - & \left\{s_{2}^{5}, s_{3}^{5}\right\} & - & \left\{s_{3}^{5}, s_{4}^{5}\right\} & \left\{s_{3}^{5}\right\} \\
\left\{s_{4}^{7}, s_{5}^{7}, s_{6}^{7}\right\} & \left\{s_{6}^{7}\right\} & - & \left\{s_{4}^{7}, s_{5}^{7}\right\} & - & - \\
\left\{s_{3}^{5}, s_{4}^{5}\right\} & - & \left\{s_{2}^{5}, s_{3}^{5}\right\} & - & \left\{s_{2}^{5}\right\} & \left\{s_{0}^{5}, s_{1}^{5}\right\} \\
\left\{s_{4}^{7}, s_{5}^{7}, s_{6}^{7}\right\} & \left\{s_{4}^{7}, s_{5}^{7}\right\} & - & \left\{s_{5}^{7}, s_{6}^{7}\right\} & - & \left\{s_{3}^{7}, s_{4}^{7}\right\} \\
\left\{s_{6}^{9}, s_{7}^{9}, s_{8}^{9}\right\} & \left\{s_{6}^{9}\right\} & - & \left\{s_{5}^{9}, s_{6}^{9}\right\} & \left\{s_{5}^{9}\right\} & -
\end{array}\right)
$$

$H^{4}=$

$$
\left(\begin{array}{cccccc}
- & \left\{s_{5}^{9}, s_{6}^{9}, s_{7}^{9}\right\} & \left\{s_{4}^{9}\right\} & \left\{s_{6}^{9}, s_{7}^{9}\right\} & \left\{s_{6}^{9}\right\} & \left\{s_{4}^{9}\right\} \\
\left\{s_{3}^{5}, s_{4}^{5}\right\} & - & \left\{s_{2}^{5}, s_{3}^{5}\right\} & - & \left\{s_{2}^{5}\right\} & \left\{s_{3}^{5}\right\} \\
\left\{s_{3}^{7}, s_{4}^{7}, s_{5}^{7}\right\} & \left\{s_{3}^{7}, s_{4}^{7}\right\} & - & \left\{s_{4}^{7}, s_{5}^{7}, s_{6}^{7}\right\} & - & - \\
\left\{s_{3}^{5}, s_{4}^{5}\right\} & - & \left\{s_{2}^{5}\right\} & - & \left\{s_{2}^{5}\right\} & \left\{s_{3}^{5}\right\} \\
\left\{s_{5}^{7}\right\} & \left\{s_{5}^{7}, s_{6}^{7}\right\} & - & \left\{s_{4}^{7}\right\} & - & \left\{s_{3}^{7}, s_{4}^{7}\right\} \\
\left\{s_{6}^{9}, s_{7}^{9}\right\} & \left\{s_{7}^{9}, s_{8}^{9}\right\} & - & \left\{s_{8}^{9}\right\} & \left\{s_{8}^{9}\right\} & -
\end{array}\right)
$$

$$
\begin{aligned}
& H^{5}= \\
& \left(\begin{array}{cccccc}
- & \left\{s_{6}^{9}, s_{7}^{9}, s_{8}^{9}\right\} & \left\{s_{5}^{9}, s_{6}^{9}\right\} & \left\{s_{6}^{9}, s_{7}^{9}\right\} & \left\{s_{5}^{9}, s_{6}^{9}, s_{7}^{9}\right\} & \left\{s_{4}^{9}, s_{5}^{9}\right\} \\
\left\{s_{3}^{5}, s_{4}^{5}\right\} & - & \left\{s_{2}^{5}\right\} & - & \left\{s_{2}^{5}, s_{3}^{5}, s_{4}^{5}\right\} & \left\{s_{2}^{5}\right\} \\
\left\{s_{5}^{7}, s_{6}^{7}\right\} & \left\{s_{4}^{7}, s_{5}^{7}\right\} & - & \left\{s_{3}^{7}, s_{4}^{7}\right\} & - & - \\
\left\{s_{3}^{5}, s_{4}^{5}\right\} & - & \left\{s_{1}^{5}, s_{2}^{5}\right\} & - & \left\{s_{4}^{5}\right\} & \left\{s_{0}^{5}, s_{1}^{5}, s_{2}^{5}\right\} \\
\left\{s_{5}^{7}, s_{6}^{7}\right\} & \left\{s_{5}^{7}\right\} & - & \left\{s_{4}^{7}, s_{5}^{7}\right\} & - & \left\{s_{4}^{7}, s_{5}^{7}\right\} \\
\left\{s_{7}^{9}, s_{8}^{9}\right\} & \left\{s_{6}^{9}, s_{7}^{9}\right\} & - & \left\{s_{6}^{9}, s_{7}^{9}, s_{8}^{9}\right\} & \left\{s_{6}^{9}, s_{7}^{9}\right\} & -
\end{array}\right)
\end{aligned}
$$

In the rest of this section, the proposed approach is employed to evaluate the collaboration ability of the six team members.

First of all, by Eq. (6), all multi-granular hesitant fuzzy linguistic evaluation matrices $H^{1}, H^{2}, \ldots, H^{5}$ are transformed into TFN evaluation matrices $Y^{1}, Y^{2}, \ldots, Y^{5}$. For instance, $Y^{1}$ is shown in the next page.

By Eqs. (7) and (8), all TFN evaluation matrices $Y^{1}, Y^{2}, \ldots, Y^{5}$ are aggregated into a collective TFN evaluation matrix $Y$, which is shown in the following pages.

By Eq. (9), the evaluation values of the six team members' collaboration ability are calculated as

$$
\begin{aligned}
& y_{1}=T(0.535,0.823,0.904,1), \\
& y_{2}=T(0.612,0.818,0.891,0.980), \\
& y_{3}=T(0.277,0.469,0.592,0.813), \\
& y_{4}=T(0.590,0.806,0.892,0.971), \\
& y_{5}=T(0.531,0.759,0.834,0.945), \\
& y_{6}=T(0.490,0.713,0.773,0.904) .
\end{aligned}
$$

Therefore, the ranking of the six team members' collaboration ability can be obtained by Eq. (3) as $P_{2} \succ P_{4} \succ P_{1} \succ P_{5} \succ P_{6} \succ P_{3}$.

Assume that the maximum number of the simple linguistic terms used by the six team members is 3 . By Eqs. (10)-(11), the evaluation values of each team member's collaboration ability is represented by HFLTSs defined on the linguistic term set used by each team member, as shown in Table 1 . Note that the results shown in Table 1 are obtained using approximation operations and may result in the loss of information to some extent.

From Table 1, one can find that based on the linguistic term set $S^{9}$ which is used by $P_{1}$ and $P_{6}$, the collaboration ability of $P_{1}, P_{2}$ and $P_{4}$ is "between good and very good", the collaboration ability of $P_{3}$ is "between slightly poor and slightly good" and the collaboration ability of $P_{5}$ and $P_{6}$ is "between slightly good and very good". Obviously, such results are interpretable and more intuitive to the team members.

\section{Conclusions}

It is of vital importance to evaluate team members' collaboration ability in an R\&D team for companies and other organizations. In this paper, a new approach is developed to evaluate the collaboration ability of R\&D team members, which allows team members to use HFLTSs defined on multi-granular linguistic term sets to elicit their linguistic assessments over team 


$$
Y^{1}=\left(\begin{array}{cccc}
T(0,0,0,0) & T(0.625,0.750,0.875,1) & T(0.5,0.625,0.75,0.875) & \\
T(0.5,0.75,0.75,1) & T(0,0,0,0) & T(0.25,0.5,0.75,1) & \\
T(0.5,0.667,0.833,1) & T(0.667,0.972,1,1) & T(0,0,0,0) & \\
T(0.75,1,1,1) & T(0,0,0,0) & T(0.25,0.5,0.5,0.75) & T(0,0,0,0) \\
T(0.5,0.667,0.8333,1) & T(0.667,0.972,1,1) & T(0,0,0,0) & \\
T(0.625,0.75,0.875,1) & T(0.625,0.914,1,1) & T(0.625,0.75,0.875,1) & T(0.375,0.5,0.625,0.75) \\
& T(0.75,0.875,0.875,1) & T(0.25,0.5,0.75,1) & T(0,0.25,0.5,0.75) \\
& T(0,0,0,0) & T(0,0,0,0) & T(0,0,0,0) \\
& T(0.333,0.633,0.7,1) & T(0.5,3.75,1,1) & T(0.25,0.5,0.75,1) \\
& T(0,0,0,0) & T(0,0,0,0) & T(0.333,0.5,0.5,0.667) \\
& T(0.5,0.852,1,1) & T(0.625,0.914,1,1) & T(0,0,0,0) \\
& T(0.75,0.984,1,1) & &
\end{array}\right.
$$

Table 1: The evaluation of each team member's collaboration ability

\begin{tabular}{ccccccc}
\hline$S^{g(i)+1}$ & $P_{1}$ & $P_{2}$ & $P_{3}$ & $P_{4}$ & $P_{5}$ & $P_{6}$ \\
\hline$S^{5}$ & $\left\{s_{3}^{5}, s_{4}^{5}\right\}$ & $\left\{s_{3}^{5}\right\}$ & $\left\{s_{2}^{5}\right\}$ & $\left\{s_{3}^{5}\right\}$ & $\left\{s_{3}^{5}\right\}$ & $\left\{s_{3}^{5}\right\}$ \\
$S^{7}$ & $\left\{s_{4}^{7}, s_{5}^{7}, s_{6}^{7}\right\}$ & $\left\{s_{5}^{7}\right\}$ & $\left\{s_{3}^{7}, s_{4}^{7}\right\}$ & $\left\{s_{5}^{7}\right\}$ & $\left\{s_{4}^{7}, s_{5}^{7}\right\}$ & $\left\{s_{4}^{7}, s_{5}^{7}\right\}$ \\
$S^{9}$ & $\left\{s_{6}^{9}, s_{7}^{9}\right\}$ & $\left\{s_{6}^{9}, s_{7}^{9}\right\}$ & $\left\{s_{3}^{9}, s_{4}^{9}, s_{5}^{9}\right\}$ & $\left\{s_{6}^{9}, s_{7}^{9}\right\}$ & $\left\{s_{5}^{9}, s_{6}^{9}, s_{7}^{9}\right\}$ & $\left\{s_{5}^{9}, s_{6}^{9}, s_{7}^{9}\right\}$ \\
\hline
\end{tabular}

members who have working relationship with them. In addition, the proposed approach not only can rank team members' collaboration ability in an R\&D team, but also can provide interpretable evaluation results for team members' collaboration ability.

In terms of future research, we intend to extend the proposed approach to evaluate team members' collaboration ability in an R\&D team based on unbalanced HFLTSs [2, 23]. It is also interesting to integrate consensus process $[3,6,27]$ into the process of collaboration ability evaluation to obtain more reasonable results.

\section{Acknowledgement}

This work was supported by the National Natural Science Foundation of China (Nos. 71501023, 71771034), the Funds for Creative Research Groups of China (No. 71421001), the China Postdoctoral Science Foundation (2015M570248), the Scientific and Technological Innovation Foundation of Dalian(2018J11CY009), the Research Funds for Young Scholars from the Education Department of Liaoning Province (LN2017QN027) and the Fundamental Research Funds for the Central Universities (DUT17RC(4)11).

\section{References}

[1] J. F. DeFranco, C. J. Neill, R. B. Clariana, A cognitive collaborative model to improve performance in engineering teams-a study of team out- comes and mental model sharing, Systems Engineering 14 (3) (2011) 267-278.

[2] Y. Dong, C.-C. Li, F. Herrera, Connecting the linguistic hierarchy and the numerical scale for the 2-tuple linguistic model and its use to deal with hesitant unbalanced linguistic information, Information Sciences 367-368 (2016) 259-278.

[3] Y. Dong, Q. Zha, H. Zhang, G. Kou, H. Fujita, F. Chiclana, E. Herrera-Viedma, Consensus reaching in social network group decision making: Research paradigms and challenges, KnowledgeBased Systems 162 (2018) 3-13.

[4] Z. Fan, B. Feng, W. Suo, A fuzzy linguistic method for evaluating collaboration satisfaction of NPD team using mutual-evaluation information, International Journal of Production Economics 122 (2) (2009) 547-557.

[5] F. Herrera, E. Herrera-Viedma, J. L. Verdegay, Direct approach processes in group decision making using linguistic owa operators, Fuzzy Sets and Systems 79 (2) (1996) 175-190.

[6] E. Herrera-Viedma, F. J. Cabrerizo, J. Kacprzyk, W. Pedrycz, A review of soft consensus models in a fuzzy environment, Information Fusion 17 (2014) 4-13.

[7] A. Kaufmann, M. M. Gupta, Introduction to Fuzzy Arithmetic: Theory and Application, Van Nostrand Reinhold, New York, 1991. 
[8] H. Leavitt, The old days, hot groups and manager's lib, Administrative Science Quarterly 41 (2) (1996) 288-300.

[9] D. Levi, S. C., Team work in research and development organizations: the characteristics of successful teams, International Journal of Industrial Ergonomics 16 (1995) 29-42.

[10] H. Liu, R. M. Rodríguez, A fuzzy envelope for hesitant fuzzy linguistic term set and its application to multicriteria decision making, Information Sciences 258 (2014) 220-238.

[11] J. MacBryde, K. Mendibil, Designing performance measurement systems for teams: theory and practice, Management Decision 41 (8) (2003) $722-733$.

[12] J. A. Morente-Molinera, I. J. Pérez, M. R. Ureña, E. Herrera-Viedma, On multi-granular fuzzy linguistic modeling in group decision making problems: A systematic review and future trends, Knowledge-Based Systems 74 (2015) 49-60.

[13] R. Rodríguez, L. Martínez, F. Herrera, Hesitant fuzzy linguistic term sets for decision making, IEEE Transactions on Fuzzy Systems 20 (1) (2012) 109-119.

[14] R. M. Rodríguez, A. Labella, L. Martínez, An overview on fuzzy modelling of complex linguistic preferences in decision making, International Journal of Computational Intelligence Systems 9 (sup1) (2016) 81-94.

[15] R. M. Rodríguez, L. Martínez, An analysis of symbolic linguistic computing models in decision making, International Journal of General Systems 42 (1) (2013) 121-136.

[16] J. Son, E. M. Rojas, Evolution of collaboration in temporary project teams: An agent-based modeling and simulation approach, Journal of Construction Engineering and Management 137 (8) (2011) 619-628.

[17] H. Wang, Z. Xu, X. Zeng, Hesitant fuzzy linguistic term sets for linguistic decision making: Current developments, issues and challenges, Information Fusion 43 (2018) 1-12.

[18] Y. Wang, H.-S. Lee, The revised method of ranking fuzzy numbers with an area between the centroid and original points, Computers and Mathematics with Applications 55 (9) (2008) 20332042.

[19] Y. Wang, J. Yang, D. Xu, K.-S. Chin, On the centroids of fuzzy numbers, Fuzzy Sets and Systems 157 (7) (2006) 919-926.
[20] C. Wei, H. Liao, A multigranularity linguistic group decision-making method based on hesitant 2-tuple sets, International Journal of Intelligent Systems 31 (6) (2016) 612-634.

[21] C. Wei, N. Zhao, X. Tang, Operators and comparisons of hesitant fuzzy linguistic term sets, IEEE Transactions on Fuzzy Systems 22 (3) (2014) 575585.

[22] X. Xu, A study on the R\&D-team building \& management, Studies in Science of Science 19 (2) (2001) $76-81$.

[23] W. Yu, Z. Zhang, Q. Zhong, L. Sun, Extended TODIM for multi-criteria group decision making based on unbalanced hesitant fuzzy linguistic term sets, Computers \& Industrial Engineering 114 (2017) 316-328.

[24] L. Zadeh, Fuzzy sets, Information and Control 8 (3) (1965) 338-353.

[25] H. Zhang, Y. Dong, X. Chen, The 2-rank consensus reaching model in the multigranular linguistic multiple-attribute group decision-making, IEEE Transactions on Systems, Man, and Cybernetics: Systems 48 (12) (2018) 2080-2094.

[26] L. Zhang, Y. Li, Q. Wu, Evaluation on collaborative satisfaction for project management team in integrated project delivery mode, Journal of The Institution of Engineers (India): Series A 94 (2) (2013) 109-115.

[27] Z. Zhang, C. Guo, Consistency and consensus models for group decision-making with uncertain 2-tuple linguistic preference relations, International Journal of Systems Science 47 (11) (2016) 2572-2587.

[28] Z. Zhang, C. Guo, L. Martínez, Managing multigranular linguistic distribution assessments in large-scale multiattribute group decision making, IEEE Transactions on Systems, Man, and Cybernetics: Systems 47 (11) (2017) 3063-3076. 